\title{
An Intelligent Conversational Agent as the Web Virtual Representative Using Semantic Bayesian Networks
}

\author{
Kyoung-Min Kim, Jin-Hyuk Hong, and Sung-Bae Cho \\ Dept. of Computer Science, Yonsei University \\ 134 Shinchon-dong, Seodaemoon-ku, Seoul 120-749, Korea \\ \{kminkim, hjinh\}@sclab.yonsei.ac.kr, sbcho@cs.yonsei.ac.kr
}

\begin{abstract}
In this paper, we propose semantic Bayesian networks that infer the user's intention based on Bayesian networks and their semantic information. Since conversation often contains ambiguous expressions, managing the context or the uncertainty is necessary to support flexible conversational agents. The proposed method drives the mixed-initiative interaction (MII) that prompts for missing concepts and clarifies for spurious concepts to understand the user's intention correctly. We have applied it to an information retrieval service for Web sites so as to verify the usefulness.
\end{abstract}

\section{Introduction}

Conversational agents are representative intelligent agents that provide information for users by using the natural language dialogue. They understand the user's intention through conversation and offer an appropriate service [1]. Pattern matching, one of the popular methods for constructing the conversational agent, works well at a sentence, but it is not feasible to understand a dialogue in which context should be considered. Moreover, it is likely to fail to understand a complex sentence which requires a deep analysis. Recently, researchers have investigated on flexible dialogue models using Bayesian networks (BN) [2], where Bayesian networks have been also used in information retrieval (IR) $[3,4]$.

When many variables in the application domain are related to each other, the inference of the user's intention becomes very difficult. In this paper, we propose semantic Bayesian networks ( $\mathrm{SeBN}$ ) not only to reduce the complexity of construction, but also to infer the user's intention in more detail.

\section{Intelligent Conversational Agent}

For the efficient inference of conversational agents, we design semantic Bayesian networks composed of the probabilistic inference and the semantic inference. The stepwise modeling helps to understand the user's intention through conversation.

It is constructed with three levels according to the function: keywords, concepts, and targets. The keyword layer consists of words related to the user's query, while the concept layer is composed of entities of the domain and their semantic relationship. 
The target layer represents target information (products) whose attributes are defined. The concept layer is divided to three components: objects, attributes, and values. Each object is a set of attribute-value pairs, where node $a_{i}$ is an attribute and node $v_{k}$ is a value in the domain. A solid line represents the probabilistic relationship between nodes, while a dotted line signifies the semantic relationship between them.

The probabilistic relationship in semantic Bayesian networks is similar to that in the traditional IR model. First, it infers probabilistically between the keyword layer and the concept layer. The user's query $U=\left\{k_{1}, k_{2}, \ldots, k_{t}\right\}$, where keyword $k_{i}$ is interpreted as an elementary word in the keyword layer. It sets a keyword node as 1 when the word of the keyword layer is observed in query $Q$ and otherwise 0 .

It infers the probability of each node in the concept layer when all evidence variables associated with keywords are set. The probability $P(c \mid W)$, using with keyword $W$ in the keyword layer as evidence, is defined as follows:

$$
\begin{aligned}
P(c \mid W) & =P\left(c \mid w_{1}, w_{2}, \ldots, w_{N}\right) \\
& =\frac{P(c) \times P\left(w_{1}, w_{2}, \ldots, w_{N} \mid c\right)}{P\left(w_{1}, w_{2}, \ldots, w_{N}\right)} \\
& \approx P(c) \times P\left(w_{1}, w_{2}, \ldots, w_{N} \mid c\right) \\
& =P(c) \times P\left(w_{1} \mid c\right) \times P\left(w_{2} \mid c\right) \times \ldots \times P\left(w_{N} \mid c\right) \\
& =P(c) \prod_{i=1}^{n} P\left(w_{i} \mid c\right)
\end{aligned}
$$

After computing the probability of all nodes in the concept layer, it infers the probability $P(p \mid C)$ of product $p$ in the target layer using them as evidence similar to that of inferring the probability $P(c \mid W)$.

Table 1. Semantic inference in SeBN

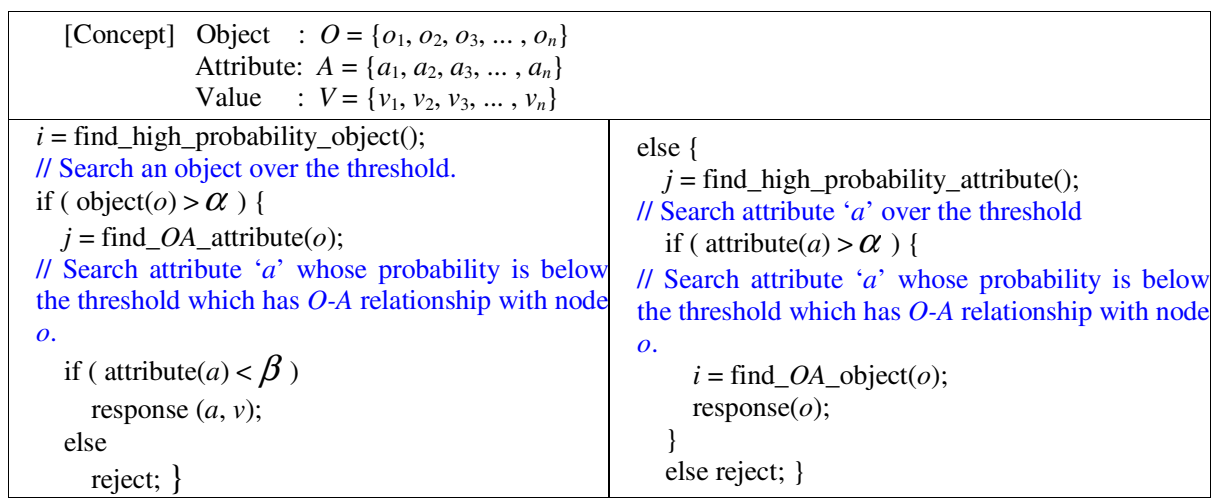

It selects a node in the target layer whose probability is higher than the threshold after the inference. It provides the information of the target product to the user when a proper number of nodes are selected. In this paper, we define it as successful execution 
when one product is selected. When there is no product selected, it executes the semantic inference of semantic Bayesian networks in the concept layer. There are two major relationships ('Has- $a$ ', ' $I s-a$ ') between nodes while ' $I s-a$ ' has two different types (' $O$ $A$ ', ' $A-V$ '). Table 1 shows the semantic inference executed when the probabilistic inference fails to infer the user's intention. At first, it searches an object node whose probability is higher than the threshold. Then, it looks up an attribute whose probability is below the threshold, which has ' $O-A$ ' relationship with the object node. It collects supplementary information on the attribute selected and carries out the inference again with the information gathered from the user. It repeats the procedure until a target product is selected.

In order to search out what the user wants, it should gather enough information to infer target products. Traditional information retrieval systems work well only when the user's query includes enough for inference. When there is not enough information, however, the proposed method provides a suitable response to the user based on the mixed-initiative interaction. Finally, the proposed method is able to show good performance in diverse dialogue situations.

\section{Experimental Results}

\subsection{Experimental Environments}

In order to verify the usefulness of the proposed method, we have developed a flexible conversational agent for virtual representative of web sites. It consists of a main window for displaying information, an input text box, and the avatar system with a speech generation engine. When the user types a query, the avatar responds in speech with a corresponding action. Q-avatar (www. qavatar.com) is employed as the avatar system, while Voiceware (voiceware.co.kr), a solution for speech generation, is used to provide the user with a realistic and convenient interface.

Table 2. The attributes of objects

\begin{tabular}{|c|l|}
\hline Object & \multicolumn{1}{|c|}{ Attributes } \\
\hline $\begin{array}{c}\text { Cellular-phone } \\
(240 \text { products })\end{array}$ & $\begin{array}{l}\text { Brand, Product, Model, Image, Bell, Camera, Pixel, Size, } \\
\text { Weight, Color, Price, Year }\end{array}$ \\
\hline $\begin{array}{c}\text { Digital camera } \\
\text { (688 products) }\end{array}$ & $\begin{array}{l}\text { Brand, Product, Model, Image, Memory, Run-time, Color, } \\
\text { Size, Feature, Weight, Price, Year }\end{array}$ \\
\hline $\begin{array}{c}\text { MP3 } \\
\text { (488 products })\end{array}$ & $\begin{array}{l}\text { Brand, Product, Model, Image, Pixel, Memory, Weight, } \\
\text { Feature, Size, Zoom, Color, Price, Year }\end{array}$ \\
\hline
\end{tabular}

The target domain is mobile Web sites introducing of cellular-phone, digital camera, and MP3. Table 2 describes the attributes of each object in the target database. The database is built by extracting information from 5 Web sites: Naver (www.nshopping.naver.com), Samsung-mall (www.samsung-mall.co.kr), LG-eshop (www.gseshop.co.kr), Enuri (www.enuri.com) and DCinside (www.dcincide. com). 


\subsection{Qualitative Analysis: Illustration}

In many cases, the user has background knowledge in addition to the content of conversation, so a query may not include all information required to infer the user's intention. The proposed conversational agent uses a mixed-initiative dialogue as shown in Fig. 1, by requesting additional information to the user. Finally, information on the target product is provided to the user after inference with the information.

As shown in Fig. 1, it searches the plural objects from the initial query. Since the agent needs additional information for the correct intention inference, it outputs a supplementary query, "What color do you like? Red or Silver?" to the user as the mixed-initiative interaction. The user responds that "I'd like a red one," and then it executes the probabilistic inference again using semantic Bayesian networks based on this response. Until it detects the plural products as the result of prior inference, the agent keeps up the conversation by the mixed-initiative interaction. If a product is selected, the agent finishes the inference and provides the information of the target product to the user.

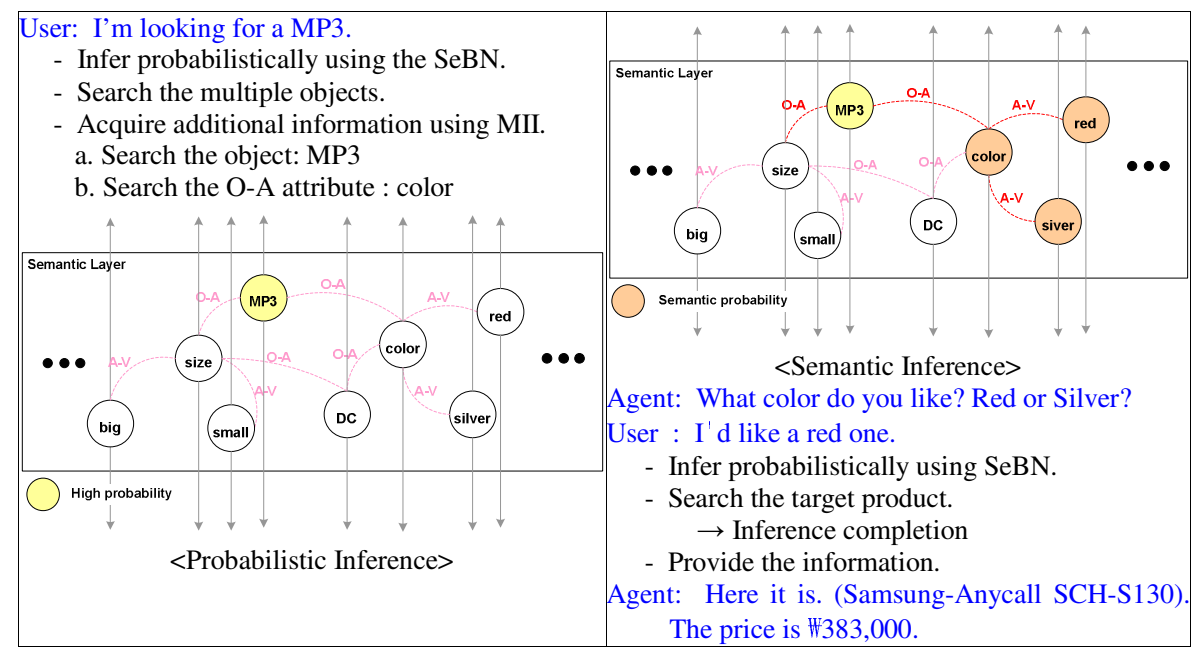

Fig. 1. The target retrieval using MII

\subsection{Quantitative Analysis}

In order to evaluate the efficacy and satisfaction of the agent by younger adults, we have compared three conversational agents: Script-based, BN-based and the SeBNbased agents. Thirty subjects in age from 22 to 33 living in Korea have evaluated them. Participants have performed ten tasks to search for information on several products. Satisfaction scores have been measured by single item on five-point Likert scales (1.0= "not at all", 5.0="very much").

The result (see Table 3 ) shows that the proposed method $(M=94.42)$ is superior to the others $(M=92.15,87.51)$. It is able to manage various types of dialogues while the 
Script-based and BN-based agents fail to respond for them. It also shows good performance in providing suitable responses for the user with few interactions $(M=2.96)$.

As shown in Table 4, satisfaction with the overall intervention has been very high in the proposed method. The effect of the proposed method on easy, friendly, informative, repetitive and interesting was statistically measured by a one way ANOVA with the variant of the SeBN as the among-systems factor. Post-hoc tests were also conducted, whenever one or more of the significant factors entail more than two categories or levels. At most cases, the proposed method shows better results than the others.

Table 3. Comparative results in efficiency

\begin{tabular}{|c|c|c|c|c|c|c|}
\hline \multirow{2}{*}{$\begin{array}{c}\text { Retrieval Rate (PR) } \\
\text { Average Interactions (AI) }\end{array}$} & \multicolumn{2}{|c|}{ Script } & \multicolumn{2}{c|}{ BN } & \multicolumn{2}{c|}{ SeBN } \\
\cline { 2 - 7 } & $\mathrm{PR}(\%)$ & $\mathrm{AI}$ & $\mathrm{PR}(\%)$ & $\mathrm{AI}$ & $\mathrm{PR}(\%)$ & $\mathrm{AI}$ \\
\hline Average & 87.51 & 3.53 & 92.15 & 3.18 & 94.42 & 2.96 \\
\hline
\end{tabular}

Table 4. Comparative results in the user satisfaction

\begin{tabular}{|c|c|c|c|c|c|c|}
\hline \multirow{2}{*}{ User Satisfaction } & \multicolumn{2}{|c|}{ Script } & \multicolumn{2}{c|}{ BN } & \multicolumn{2}{c|}{ SeBN } \\
\cline { 2 - 7 } & Mean & SD & Mean & SD & Mean & SD \\
\hline Easy & 2.9 & .7379 & 4.0 & .4714 & 4.6 & .5164 \\
\hline Friendly & 2.7 & .6749 & 3.8 & .4216 & 4.7 & .4830 \\
\hline Informative & 3.1 & .5676 & 3.7 & .6749 & 4.4 & .5164 \\
\hline Repetitive & 3.9 & .8756 & 2.3 & .4830 & 1.6 & .5164 \\
\hline Interesting & 3.1 & .5676 & 3.8 & .7888 & 4.5 & .5270 \\
\hline
\end{tabular}

\section{Conclusion and Future Works}

We have proposed a conversational agent using semantic Bayesian networks to be more flexible and considerable in the inference of the intention. If information in the query is insufficient, the agent asks the user to give more information to infer the user's intention correctly. Finally, it improves the answering performance. While, designing networks becomes easier and more comprehensible by one's intuition. The research on the automatic construction of semantic networks is remained for the future study.

\section{Acknowledgements}

The work was supported by the Korea Research Foundation Grant. (KRF-2004-005$\mathrm{H} 00005$ ) 


\section{References}

1. S. Macskassy, "A conversational agent," Master Essay, Rutgers University, 1996.

2. J.-H. Hong and S.-B. Cho, "A two-stage Bayesian network for effective development of conversational agent," LNCS 2690, pp. 1-8, 2003.

3. S. Acid, et al., "An information retrieval model based on simple Bayesian networks," Int. Journal of Intelligent Systems, 18(2), pp. 251-265, 2003.

4. P. Calado, et al., "A Bayesian network approach to searching Web databases through keyword-based queries," Information Processing and Management, 40, pp. 773-790, 2004. 